\title{
Direct Imaging of Rhodium Crystal Surface Structures with Signal Recovery by Low Dose Microscopy
}

\author{
P. Specht, ${ }^{*}$ D. Barton, ${ }^{* *}$ J. Kang, ${ }^{* *}$ R.C. Cieslinski, ${ }^{* *}$ O.D. Dubon, ${ }^{*}$ C. Kisielowski*** \\ * University of California, Dept. of Materials Science and Engineering, and Materials Science \\ Division, Lawrence Berkeley National Laboratory, One Cyclotron Rd., Berkeley CA94720 \\ ** Dow Chemical Company, Midland, MI 48642 \\ *** National Center for Electron Microscopy and Helios SERC, Lawrence Berkeley National \\ Laboratory, One Cyclotron Rd., Berkeley CA 94720
}

Aberration-corrected TEM as well as the development of monochromators and high brightness guns have advanced transmission electron microscopy to a point where resolution is no longer a limiting process in imaging atomic structures. Instead, beam-sample interactions become increasingly important, particularly for nano-structures and light elements. Recently it was found that phononinduced atom vibrations significantly change the image of a rhodium nano-cluster under electron beam illumination [1]. A characteristic intensity fluctuation could be described by this phenomenon. Following, a low dose phase contrast imaging technique is applied which minimizes beam-sample interactions including phonon-induced atom vibrations and therefore results in more complete data acquisition with improved signal strength.

Nanostructures such as ultrathin sheets such as graphene, nanowires and nano-sized clusters such as catalyst materials are due to their limited dimensions more prone to beam-sample interactions than bulk crystals. Such interactions can result in phonon-induced atom motion, diffusion or loss of surface atoms [2] or even phase changes of a whole cyrstal. The original structure of nanostructures may be masked by such processes and their crystallography and atomic arrangement on the surfaces remains inaccessible. It is shown that low dose microscopy allows for a reduction of beam-induced sample alterations while maintaining atomic resolution, providing higher signals and giving access to surface structures which remain unresolved under high beam conditions.

Fig. 1 gives a comparison of two single images taken in a TEAM0.5 microscope at $80 \mathrm{kV}$ acceleration voltage, $\mathrm{C}_{\mathrm{S}}$ corrected with $\mathrm{C}_{\mathrm{S}}=-15 \mu \mathrm{m}$ and utilizing a monochromated high brightness (XFEG) beam. On the left a structural image taken with a dose rate of approx. $5000 \mathrm{e}^{-/} /\left(\AA^{2}\right.$ s) shows a rhodium crystal on alumina support. On the right the surfaces of the rhodium cluster are barely visible due to the low dose applied of approx. $75 \mathrm{e}^{-} /\left(\AA^{2} \mathrm{~s}\right)$. Low dose conditions were achieved by using the monochromated beam plus a slit which illuminates an area of $80 \mathrm{~nm} \times 80 \mathrm{~nm}$, the dose rate can be changed by changing the potential of the monochromator. Details are described in [3]. Figure 2 displays the phase of the exit wave which was reconstructed from focal series for both dose conditions. Focal series were taken with $0.5 \mathrm{~nm}$ change in focus, recording 100 images. The corresponding line scans (bottom of Fig. 2) show that the phase range for the low dose phase image is increased by $20 \%$. At the surface of the rhodium cluster additional atomic features can be resolved (arrow). It is shown that low dose microscopy improves the structural identification of nanostructures [4].

[1] P. Specht et al., ChemCatChem. (2011) in review.

[2] P. Specht et al., Microsc. Microanal. 16 (2010) 122-123 
[3] D. Detert et al., Microsc. Microanal. (2011) submitted

[4] Work was supported by DOW Chemical Comp. under contract no. M80036. TEM was done at the National Center for Electron Microscopy (NCEM) of LBNL, supported by the Director, Office of Science, Office of Basic Energy Sciences of the U.S. Department of Energy under Contract No. DEAC02-05CH11231
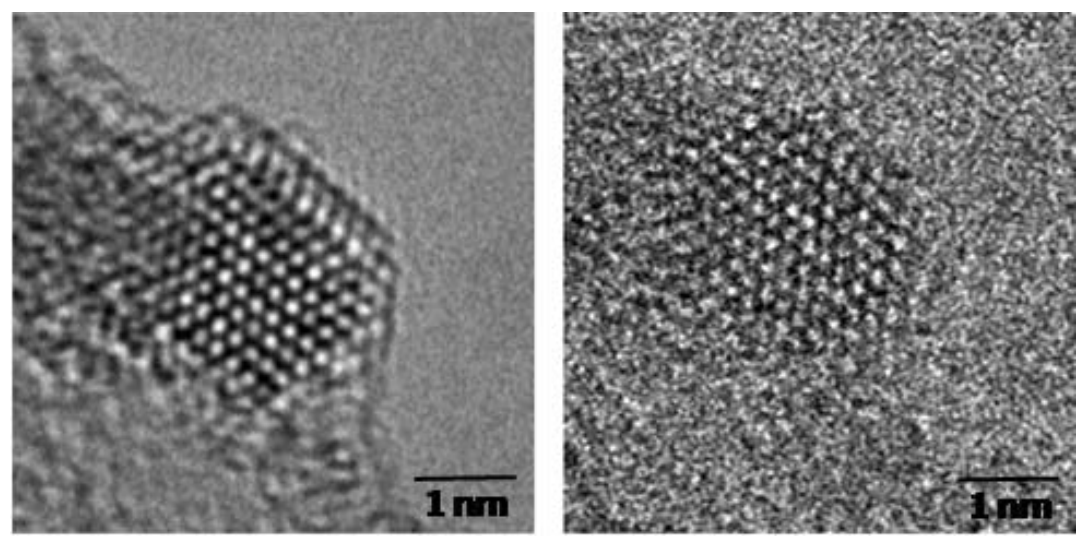

FIG. 1. Phase contrast image of rhodium particle on alumina support at $80 \mathrm{kV}$ acceleration voltage. Left: dose rate about $5000 \mathrm{e}^{-/}\left(\AA^{2} \mathrm{~s}\right)$, right: dose rate about $75 \mathrm{e}^{-} /\left(\AA^{2} \mathrm{~s}\right)$. Atom columns are visible in both structural images
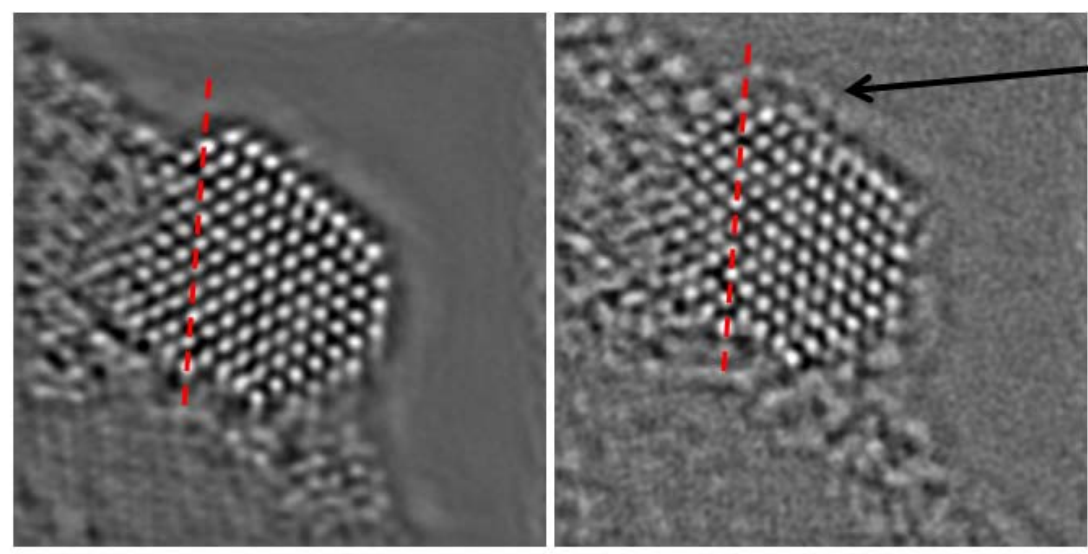

linescan

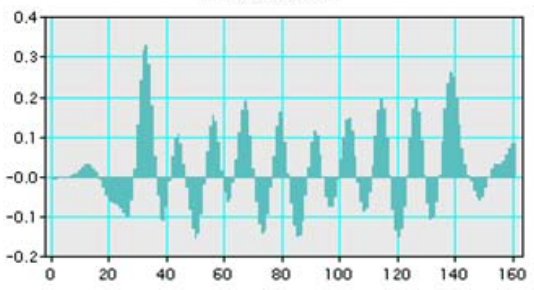

linescan

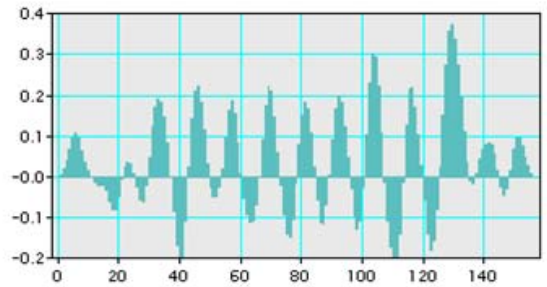

FIG. 2. Focal series reconstructions of rhodium particle for both dose rates in fig. 1. The line scans (bottom figures) are taken at the dashed lines in the figures above, the low dose image (right) shows additional features at the surface of the particle (see arrow). Acceleration voltage: $80 \mathrm{kV}, \mathrm{C}_{\mathrm{s}}=-15 \mu \mathrm{m}$ 